\title{
The European Joint Programme on Radioactive Waste Management EURAD: update of its strategic research agenda
}

\author{
Dirk Bosbach $^{1}$, Crina Bucur ${ }^{2}$, and Christophe Bruggeman ${ }^{3}$ \\ ${ }^{1}$ Institut für Energie und Klimaforschung, Forschungszentrum Jülich, Jülich, Germany \\ ${ }^{2}$ Institute for Nuclear Research, Pitesti, Romania \\ ${ }^{3}$ SCK CEN, Brussels, Belgium \\ Correspondence: Dirk Bosbach (d.bosbach@fz-juelich.de) \\ Published: 10 November 2021
}

\begin{abstract}
The European Joint Programme on Radioactive Waste Management EURAD brings together various research actors, namely waste management organisations (WMO), technical support organisations (TSO) and research entities (RE), to work on a joint strategic research agenda (SRA) focusing on deep geological disposal of radioactive waste. In total, 116 project partners from 23 countries have worked jointly since 2019 in collaborative $R D \& D$ work packages, strategic studies and various knowledge management activities. EURAD research is driven by the need for implementation of a deep geological repository and its safety, while aiming for scientific excellence. EURAD has developed a roadmap which is seen as a representation of a generic radioactive waste management (RWM) programme. The content is focused on what knowledge and competencies (including infrastructures) are considered most critical for RWM and implementation of deep geological disposal, in alignment with the EURAD vision.

Here, the current SRA update process will be outlined from the perspective of Europe's research entities contributing to EURAD. In this context, the international network of research entities EURADSCIENCE plays a key role. EURADSCIENCE addresses - and will address during decades to come - scientific excellence in (the full lifecycle of) radioactive waste management from cradle to grave. As an independent, cross-disciplinary and inclusive organization, its overarching aim is to ensure scientific excellence and credibility in decision-making on RWM, regardless of national implementation status, waste type or national inventory. To this end, EURADSCIENCE will define and update its own SRA. The approach here is to maintain a holistic view of scientific disciplines and provide scientific excellence to advance progress of national radioactive waste management programmes, and to ensure scientific credibility of waste management concepts as well as addressing fundamental requirements related to knowledge management. More generally speaking, EURADSCIENCE aims to bring forward a vision that assures that scientific excellence and ever-developing scientific advances are integrated at any given time into the multigenerational implementation process of geological disposal.

Similarly, the respective WMO and TSO networks, IGD-TP and SITEX, have developed their SRAs based on their specific roles and perspectives. Ultimately, the overlap between these SRAs will define the envelope for future European RD\&D activities in the context of RWM. The update process has recently been consolidated after consultations between the three actor groups. Ultimately, the EURAD general assembly will have to approve the SRA update process regarding its alignment with the EURAD roadmap, the development of the seven existing SRA themes, the development of future RD\&D activities via an EURAD exchange forum and the focus of RD\&D planning for the next 10 years.
\end{abstract}

Kurzfassung. Das gemeinsame Forschungsprogramm der Europäischen Union zu Entsorgung und Management radioaktiver Abfälle EURAD bringt verschiedene Forschungsakteure zusammen, darunter Abfallentsorger (Waste Management Organisations - WMO), Sachverständigenorganisation (Technical Support Organisations 
- TSO) und Forschungseinrichtungen (Research Entities - RE), um an einer gemeinsamen strategischen Forschungsagenda mit Schwerpunkt auf der geologischen Tiefenlagerung radioaktiver Abfälle zu arbeiten. Insgesamt 116 Projektpartner aus 23 Ländern arbeiten seit 2019 in gemeinsamen F\&E-Arbeitspaketen, strategischen Studien und verschiedenen Wissensmanagementaktivitäten zusammen. Die EURAD-Forschung orientiert sich an den Erfordernissen der Einrichtung eines geologischen Tiefenlagers und dessen Sicherheit, wobei wissenschaftliche Exzellenz angestrebt wird. EURAD hat eine Roadmap entwickelt, die als Darstellung eines generischen Programms für die Entsorgung radioaktiver Abfälle zu verstehen ist. Der Inhalt konzentriert sich auf die Kenntnisse und Kompetenzen (einschließlich Infrastruktur), die für Entsorgung radioaktiver Abfälle und die Einrichtung geologischer Tiefenlagerung als besonders wichtig erachtet werden, und orientiert sich an der EURAD-Vision.

Hier wird der derzeitige Aktualisierungsprozess der Strategischen Forschungsagenda aus der Perspektive der europäischen Forschungseinrichtungen, die einen Beitrag zu EURAD leisten, skizziert. In diesem Zusammenhang spielt das internationale Netzwerk von Forschungseinrichtungen EURADSCIENCE eine Schlüsselrolle. EURADSCIENCE befasst sich heute und in den kommenden Jahrzehnten mit wissenschaftlicher Exzellenz bei (dem gesamten Lebenszyklus) der Entsorgung radioaktiver Abfälle, von der Wiege bis zur Bahre. Als unabhängige, interdisziplinäre und inklusive Organisation besteht ihr übergeordnetes Ziel darin, wissenschaftliche Exzellenz und Glaubwürdigkeit bei der Entscheidungsfindung im Bereich der Entsorgung radioaktiver Abfälle zu gewährleisten, unabhängig vom nationalen Umsetzungsstatus, der Abfallart oder dem nationalen Inventar. $\mathrm{Zu}$ diesem Zweck wird EURADSCIENCE seine eigene Strategische Forschungsagenda definieren und aktualisieren. Der Ansatz besteht darin, eine Gesamtsicht der wissenschaftlichen Fachrichtungen einzunehmen und wissenschaftliche Exzellenz zu erreichen, um den Fortschritt der nationalen Programme zur Entsorgung radioaktiver Abfälle voranzutreiben und die wissenschaftliche Glaubwürdigkeit von Abfallentsorgungskonzepten zu gewährleisten sowie grundlegende Anforderungen im Zusammenhang mit Wissensmanagement zu berücksichtigen. Allgemeiner ausgedrückt, will EURADSCIENCE eine Vision vorantreiben, die sicherstellt, dass wissenschaftliche Spitzenleistungen und sich ständig weiterentwickelnde wissenschaftliche Fortschritte zu jedem Zeitpunkt in den generationenübergreifenden Umsetzungsprozess der geologischen Endlagerung integriert werden.

In ähnlicher Weise haben die jeweiligen WMO- und TSO-Netzwerke, IGD-TP und SITEX, ihre Strategischen Forschungsagenden auf der Grundlage ihrer spezifischen Rollen und Perspektiven entwickelt. Letztlich wird die Überschneidung dieser Strategischen Forschungsagenden den Rahmen für künftige europäische F\&EAktivitäten im Zusammenhang mit der Entsorgung radioaktiver Abfälle festlegen. Der Aktualisierungsprozess wurde kürzlich nach Konsultationen zwischen den drei Akteursgruppen konsolidiert. Letztendlich muss die EURAD-Generalversammlung den Aktualisierungsprozess der Strategischen Forschungsagenden in Bezug auf die Anpassung an die EURAD-Roadmap, die Entwicklung der sieben bestehenden Themen der Strategischen Forschungsagenda, die Entwicklung künftiger F\&E-Aktivitäten über ein EURAD-Austauschforum und den Schwerpunkt der F\&E-Planung für die nächsten 10 Jahre genehmigen. 